\title{
Clinical Use of a Multivariate Electroencephalogram (Narcotrend) for Assessment of Anesthetic Depth in Horses during Isoflurane-Xylazine Anesthesia
}

OPEN ACCESS

Edited by:

Karine Portier,

University Claude Bernard Lyon 1,

France

Reviewed by:

Keila Ida,

Université de Liège, Belgium

Miguel Gozalo Marcilla,

Universidade Estadual Paulista

"Júlio de Mesquita Filho", Brazil

*Correspondence:

Sabine B. Kästner

sabine.kaestner@tiho-hannover.de

tPresent address:

Sabine B. Kästner,

Center for Systems Neuroscience Hannover, University of Veterinary Medicine Hannover Foundation, Hannover, Germany

Specialty section:

This article was submitted to Veterinary Surgery and Anesthesiology,

a section of the journal

Frontiers in Veterinary Science

Received: 03 November 2015

Accepted: 04 March 2016

Published: 17 March 2016

Citation:

Tünsmeyer J, Hopster K and Kästner SB (2016) Clinical Use of a Multivariate Electroencephalogram (Narcotrend) for Assessment of Anesthetic Depth in Horses during Isoflurane-Xylazine Anesthesia. Front. Vet. Sci. 3:25. doi: 10.3389/fvets.2016.00025

\author{
Julia Tünsmeyer ${ }^{1}$, Klaus Hopster ${ }^{2}$ and Sabine B. Kästner, ${ }^{1,2 *}$ \\ ${ }^{1}$ Small Animal Clinic, University of Veterinary Medicine Hannover Foundation, Hannover, Germany, ${ }^{2}$ Equine Clinic, University \\ of Veterinary Medicine Hannover Foundation, Hannover, Germany
}

Objective: To investigate the use of the Narcotrend electroencephalogram (EEG) monitor for the assessment of anesthetic depth in horses undergoing xylazine balanced isoflurane anesthesia.

Study design: Blinded experimental study.

Animals: Seven healthy warm-blooded horses, aged $10.6 \pm 5.9$ years, weighing $535 \pm 55 \mathrm{~kg}$.

Methods: Horses were anesthetized for a terminal surgical trial with xylazine, thiopentone, and guaiphenesin for induction and isoflurane and xylazine continuous rate infusion for maintenance. After surgery, an EEG was recorded and processed by the Narcotrend monitor. It displays an index [Narcotrend index (NI)] between 0 and 100, which is supposed to indicate anesthetic depth. This index was recorded and correlated with eight different end tidal (ET) isoflurane concentrations between 0.8 and 2.2 vol\%. In addition, anesthetic depth was numerically scored based on common clinical signs with a score of 1 (plane "too deep") to 4 (plane "too light"). After testing for normal distribution, both clinical scores and $\mathrm{NI}$ were correlated with different ET isoflurane concentrations using Spearman rank correlation.

Results: Correlation of NI with ET isoflurane concentrations was poor $\left(r_{s}=0.24\right)$. The $\mathrm{NI}$ ranged between maximal 48 and minimal 13 in the horses. The clinical scores decreased with increasing ET isoflurane concentrations $\left(r_{\mathrm{s}}=0.80\right)$. They ranged from 1 to 4 in different horses at the concentrations investigated.

Conclusion: In this study, the NI did not seem to be useful for assessment of anesthetic depth in horses receiving isoflurane anesthesia balanced with a xylazine constant rate infusion.

Keywords: horses, EEG, Narcotrend, anesthesia, isoflurane 


\section{INTRODUCTION}

The exact determination of anesthetic depth is still a challenge, and the electroencephalogram (EEG) has been extensively investigated as a tool to monitor anesthetic depth in human beings and also in horses. Precise assessment of central nervous system (CNS) activity in horses is of particular importance because too light anesthetic planes may be associated with movement, leading to injury of the horse or personnel. Too deep anesthetic planes increase the risk of complications, such as cardiorespiratory depression and postanesthetic myopathy $(1,2)$. This might be one reason for the high perioperative mortality rate in this species compared to small animals $(3,4)$.

Different studies investigated EEG power spectrum analysis in horses $(5,6)$. However, in human medicine, this technique has more and more been replaced by the use of multivariate monitors using algorithms, such as the bispectral index (BIS). In humans, BIS seems to be a reliable indicator of anesthetic depth with different anesthetic protocols (7). Similarly, in veterinary medicine, BIS was inversely correlated with inhalation anesthetic concentrations in dogs, cats, and pigs (8-10). However, in isofluraneanesthetized horses, BIS did not precisely indicate the degree of CNS depression (11).

The Narcotrend is a different multivariate EEG monitor, which uses algorithms based on a visual classification of the human sleep EEG, classical spectral parameters, and algorithms for recognition of suppression lines (12). In the same way as BIS, it displays anesthetic depth by a dimensionless index between 0 (electrical silence) and 100 (awake). Multiple human clinical and validation studies investigating the Narcotrend are available, including comparison of Narcotrend to BIS, with good results $(13,14)$. Nevertheless, veterinary experience is limited $(15,16)$. In dogs anesthetized with inhalant agents, Narcotrend index (NI) differed between anesthetic planes assessed clinically as "too deep" and "adequate," but not between planes assessed as "too light" and "adequate" (15).

To the best of our knowledge, no published data are available about the Narcotrend assessing anesthetic depth in horses. Therefore, the aim of this study was to investigate if the NI correlates with different end tidal (ET) isoflurane concentrations in horses and to compare this correlation to common clinical signs that are routinely used to monitor anesthetic depth in horses.

We hypothesized that the NI would be able to detect different stages of anesthesia reflected by different ET isoflurane concentrations.

\section{MATERIALS AND METHODS}

Seven warm-blooded horses, aged $10.6 \pm 5.9$ years, weighing $535 \pm 55 \mathrm{~kg}$, were anesthetized for a terminal experimental abdominal surgery approved by the local animal welfare committee (number 33.14-42502-04-11/0572). The EEG part of the study was started after termination of the abdominal surgery, because it was not possible to vary anesthetic depth during the surgical study. The surgeries lasted approximately $2 \mathrm{~h}$. After investigating the NI at different ET isoflurane concentrations, the horses were euthanized with $80 \mathrm{mg} \mathrm{kg}^{-1}$ pentobarbital, and the cadavers were used for anatomical studies.

Prior to anesthesia, all the horses were assessed to be healthy by physical examination and complete blood cell count (American Society of Anesthesiologists classification I/ II). All the horses were premedicated intravenously (IV) with xylazine (Xylapan, Vetoquinol, Germany) until deeply sedated $\left(0.8-1.1 \mathrm{mg} \mathrm{kg}^{-1}\right)$ and placed in stocks with a swinging door. Guaiphenesin (Myolaxin15\%, Vetoquinol, Germany) was infused IV to effect $\left(75-100 \mathrm{mg} \mathrm{kg}^{-1}\right)$ until horses became ataxic, and anesthesia was induced with $5 \mathrm{mg} \mathrm{kg}^{-1}$ thiopental IV (Trapanal, Nycomed Deutschland GmbH, Germany). Following orotracheal intubation (Cuffed endotracheal silicone tube, Smiths Medical ASD Inc., Germany; ID $25-30 \mathrm{~mm}$ ), horses were hoisted on the surgical table, placed in dorsal recumbency, and connected to a large animal circle system. Anesthesia was maintained with isoflurane (Isofluran $\mathrm{CP}, \mathrm{CP}$-Pharma, Germany) delivered in $4-81 \mathrm{~min}^{-1}$ of $100 \%$ oxygen $\left(\mathrm{O}_{2}\right)$, and a xylazine infusion at a continuous rate of $0.75 \mathrm{mg} \mathrm{kg}^{-1} \mathrm{~h}^{-1}$ delivered by a syringe driver (Braun Perfusor Compact S, B. Braun Melsungen AG, Germany), which was started immediately after induction.

Lactated Ringer's solution (Ringer-Laktat-Lösung, B. Braun Melsungen AG, Germany) was administered at a rate of $10 \mathrm{ml} \mathrm{kg} \mathrm{k}^{-1} \mathrm{~h}^{-1}$, and dobutamine (Dobutamin-ratiopharm $250 \mathrm{mg}$, ratiopharm $\mathrm{GmbH}$, Germany) $\left(0.5 \mu \mathrm{g} \mathrm{kg}^{-1} \mathrm{~min}^{-1}\right)$ was administered via syringe driver (Braun Perfusor Compact S, B. Braun Melsungen AG, Germany) to maintain mean arterial blood pressure (MAP) above $60 \mathrm{mmHg}$. A MAP below $60 \mathrm{mmHg}$ was defined as hypotension. For the evaluation of anesthetic depth, a MAP $>90 \mathrm{mmHg}$ was presumed as "elevated," a MAP $<90$ and $>70 \mathrm{mmHg}$ as "normal," a MAP $<70$ and $>65 \mathrm{mmHg}$ as "minimally depressed," a MAP $<65$ and $>60 \mathrm{mmHg}$ as "moderately depressed," and a MAP $<60 \mathrm{mmHg}$ as "markedly depressed" (Table 1).

TABLE 1 | Clinical scoring system of anesthesia depth in horses [modified from Hubbell and Muir (17)].

\begin{tabular}{|c|c|c|c|c|}
\hline $\begin{array}{l}\text { Anesthetic } \\
\text { plane }\end{array}$ & $\begin{array}{l}\text { Clinical } \\
\text { score }\end{array}$ & Pupil position/size & $\begin{array}{l}\text { Eye reflex ( } P \text {, palpebral; } \\
C \text {, corneal) }\end{array}$ & Heart rate/blood pressure/(spontaneous respiration) \\
\hline Too light & 4 & Central/large or small & P: active; C: active & Normal or elevated/breathing against ventilator; occasional swallowing \\
\hline Medium & 3 & Ventromedial/small or medium & P: depressed; C: mildly depressed & Normal or minimally depressed \\
\hline Medium-deep & 2 & Central/medium & P: depressed; C: depressed & Minimally to moderately depressed \\
\hline Too deep & 1 & Central/large & P: absent; C: markedly depressed & Markedly depressed \\
\hline
\end{tabular}

A mean arterial pressure (MAP) >90 mmHg was presumed as "elevated," a MAP $\leq 90$ and $\geq 70$ mmHg as "normal," a MAP <70 and $\geq 65$ mmHg as "minimally depressed," a MAP $<65$ and $>60 \mathrm{mmHg}$ as "moderately depressed," and a MAP $\leq 60 \mathrm{mmHg}$ as "markedly depressed".

A heart rate $(H R)>45$ beats per minute $(\mathrm{bpm})$ was supposed to be elevated, a HR between 30 and 45 bpm was thought to be normal, and a HR <30 bpm was thought to be depressed. 
Pressure-cycled intermittent positive pressure ventilation (Bird Mark 7, Vet.-Tec. Model JAVC 2000 J.D. Medical Distributing Company, Phoenix, AZ, USA) was initiated immediately after connection to the anesthetic machine in all horses and adjusted to maintain end tidal carbon dioxide (ET $\mathrm{CO}_{2}$ ) between 4.7 and $6 \mathrm{kPa}(35-45 \mathrm{mmHg})$. The transverse facial artery was cannulated with a $21-\mathrm{G}$ catheter (Introcan-W, B. Braun Melsungen AG, Germany) for invasive blood pressure monitoring and arterial blood sampling. The catheter was connected to a pre-calibrated pressure transducer (Gould Statham Transducer, PD 23 ID, USA) via fluid-filled, non-compliant extension lines, and the pressure transducer was positioned at the level of the shoulder joint and zeroed to atmospheric pressure. Respiratory gases were sampled continuously from the $y$-piece. Electrocardiogram (ECG), respiratory rate, inspiratory and ET $\mathrm{CO}_{2}, \mathrm{O}_{2}$ and isoflurane concentrations, $\mathrm{O}_{2}$ saturation of hemoglobin via pulse oximetry $\left(\mathrm{SpO}_{2}\right)$, and arterial blood pressure were monitored continuously with a multiparametric anesthesia monitor (Kardiocap 5 monitor, Datex-Ohmeda GmbH, Germany) and recorded every $10 \mathrm{~min}$. Arterial blood gases were sampled every $30 \mathrm{~min}$ and immediately analyzed (AVL995, AVL Medizintechnik, Germany).

The Narcotrend monitor (Monitor Technik, Bad Bramstedt, Germany) was used for recording and processing of the raw EEG. The raw EEG signal was recorded by standard silver ECG needle electrodes using a single-channel registration. The recording electrodes were subcutaneously placed modified from Mayhew and Washbourne (18), with the active electrodes bilaterally placed over the zygomatic processes and the reference placed caudal to the poll. Correct needle placement was checked by automatic impedance measurements of the monitor. The positions of needles were changed when the impedance was $6 \mathrm{k} \Omega$ or higher. The EEG was continuously recorded and processed to the Narcotrend stages and indices, and the data were stored by the monitor for off-line analysis.

The Narcotrend monitor analyzes the EEG signal in epochs of $20 \mathrm{~s}$, and the display is updated every $5 \mathrm{~s}$. The monitor displays an anesthetic stage between A (awake) and F (very deep hypnosis), which is further subdivided in the NI ranging between 100 (awake) and 0 (isoelectricity). Due to greater precision and better comparability to other EEG monitors, such as the BIS, only the NI was analyzed in this study.

Randomly assigned by drawing labeled papers from a box to either an ascending or a descending order, vaporizer settings were adjusted to achieve eight different ET isoflurane concentrations $(0.8,1.0,1.2,1.4,1.6,1.8,2.0$, and 2.2 vol\%). The observer assessing anesthetic depth by clinical signs was blinded to the vaporizer settings and the EEG monitor readings. ET concentrations were kept constant for an equilibration phase of $15 \mathrm{~min}$. After $15 \mathrm{~min}$, the NI was recorded, and anesthetic depth was assessed clinically using a score between 1 ("too deep" plane) and 4 ("too light" plane), with a score of 2 reflecting a "mediumdeep" anesthetic plane and a score of three a "medium" plane (Table 1). If horses were between two planes based on their clinical parameters, half scores were recorded. For the clinical score adapted from Hubbell and Muir (17), spontaneous movement led automatically to the evaluation as "too light." For possible major movement, a thiopentone bolus of $5 \mathrm{mg} \mathrm{kg}^{-1}$ was prepared for IV injection.

\section{Statistical Analysis}

The Kolmogorov-Smirnov test and histograms were used for evaluation of normal distribution. Correlation was analyzed by Spearman rank correlation coefficient. A $p<0.05$ was considered significant.

\section{RESULTS}

Vital parameters ranged in clinical acceptable limits for anesthetized horses in all individuals during anesthesia. None of the horses experienced severe hypotension ( $\mathrm{MAP}<60 \mathrm{mmHg}$ ), hypoxemia $\left(\mathrm{PaO}_{2}<10.7 \mathrm{kPa}\right)(<80 \mathrm{mmHg})$, severe hypercapnia $\left(\mathrm{PaCO}_{2}>8 \mathrm{kPa}\right)(>60 \mathrm{mmHg})$, hypocapnia $\left(\mathrm{PaCO}_{2}<4 \mathrm{kPa}\right)$ $\left(<30 \mathrm{mmHg}\right.$ ), or hypothermia (rectal temperature $<34^{\circ} \mathrm{C}$ ). Therefore, it was not necessary to increase the dobutamine infusion rate in any of the horses. During the study, none of the horses showed movement requiring thiopentone injection.

Over a wide range of lower ET isoflurane concentrations, the NI did not change considerably. Only at high ET concentrations of 2.0 and $2.25 \mathrm{vol} \%$, a sudden decrease of the NI was observed in four of seven horses (Figure 1), and the correlation between $\mathrm{NI}$ and isoflurane concentration was poor $\left(r_{\mathrm{s}}=0.24 ; p=0.078\right)$. However, the clinical score decreased continuously with increasing isoflurane concentrations (Figure 2), and correlation was $\operatorname{good}\left(r_{s}=0.80 ; p<0.001\right)$.

\section{DISCUSSION}

In the present study, NI did not change over a wide range of isoflurane concentrations in all of the seven horses. In contrast to the clinical evaluation, NI did not detect a decrease in anesthetic depth at the lower ET isoflurane concentrations. Only at very high isoflurane concentrations (2.0-2.2 vol\%), a decrease in NI was observed.

The results presented here are in accordance with an investigation about a different multivariate EEG monitor, the BIS,

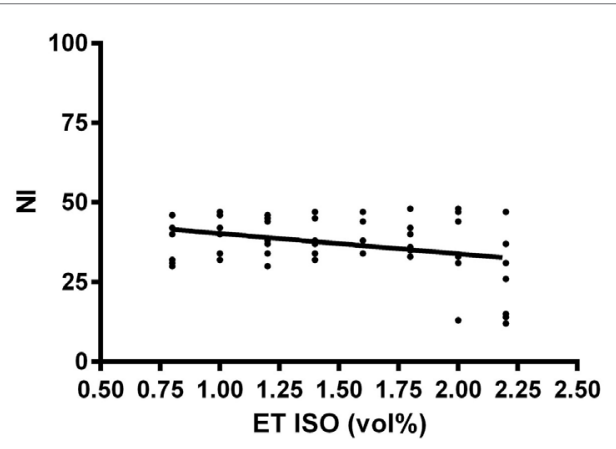

FIGURE 1 | Relationship of the dimensionless Narcotrend index (NI) with eight different end tidal isoflurane concentrations (ET ISO) in volume percent (vol\%) after an equilibration phase of $15 \mathrm{~min}$ in seven horses. The bold line included in the scatter plot is the regression line. 


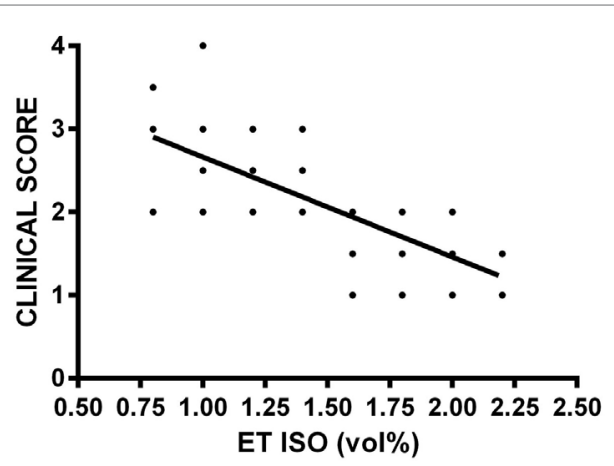

FIGURE 2 | Relationship of a clinical score between 1 and 4 evaluating anesthetic depth with eight different end tidal isoflurane concentrations (ET ISO) in volume percent (vol\%) after an equilibration phase of $\mathbf{1 5} \mathbf{~} \mathbf{m i n}$ in seven horses. The clinical score is based on common physical signs of anesthetic depth, such as eye reflexes, pupil size and position, respiration, heart rate, and arterial blood pressure [modified from Hubbell and Muir (17)], with a score of 4 reflecting a "too light" anesthetic plane, score of 3 reflecting a "medium" anesthetic plane, score of 2 reflecting a "medium-deep" anesthetic plane, and score of 1 reflecting a "too deep" anesthetic plane. The bold line included in the scatter plot is the regression line.

in horses (11). In this study, BIS was not able to distinguish between the sedated and the anesthetized state and paradoxically increased at an ET isoflurane concentration of 1.9 vol\% when compared to $1.4 \mathrm{vol} \%$. In contrast, in a study in horses receiving halothane or sevoflurane anesthesia, BIS detected a difference in CNS depression between anesthetized and awake horses (19).

One explanation for these conflicting results could be a specific effect of isoflurane on the equine EEG. In a study investigating the effects of volatile anesthetics on EEG spectral parameters, an increase in ET isoflurane concentration from 1.25 to $1.5 \mathrm{MAC}$ paradoxically increased spectral edge frequency of the equine EEG (19). In a different study, isoflurane similarly increased spectral edge frequency, whereas halothane and methoxyflurane reduced it with increasing concentrations (20). In a recent experimental study about the effects of monoanesthetics with different volatile agents on the equine EEG, none of the quantitative EEG data provided useful information to monitor anesthesia and offered little advantage over the use of changes in MAP for this purpose (21). The BIS reversely increased with increasing isoflurane MAC levels in that study (21). However, when propofol was used instead of volatile agents for anesthetic maintenance in horses, BIS was also not different between multiples of the propofol minimum infusion rate (MIR) in anesthesia and correlation with MIR was only moderate (22).

Another possible explanation for the poor correlation in our study is that multivariate monitors, such as Narcotrend, have been developed for use in humans. For example, the Narcotrend incorporates algorithms based on the human sleep EEG, which may not completely be transferable to other species. In dogs anesthetized with isoflurane or sevoflurane, similar to the horses reported here, NI was not different between anesthetic planes assessed clinically as "too light" and as "adequate" but differentiated those planes from a clinical evaluation as "too deep" (15). The difficulty of evaluating the use of such EEG monitors is the lack of some "gold standard" reference method, which determines the hypnotic component of anesthesia precisely. In veterinary anesthesia, commonly used variables include reflex response, muscle tone, respiratory, and hemodynamic parameters, which only indirectly reflect the hypnotic state of a patient.

Clinical evaluation of anesthetic depth seemed to be superior to NI in the examined horses due to better correlation with isoflurane concentrations and differentiation between a "too light" and an "adequate" plane of anesthesia. However, the loss of reflex movement is usually due to inhibition of sensory or motor pathways at the segmental level of spinal cord or brainstem $(23,24)$, and isoflurane has been shown to inhibit spinal motor neurons and produce immobility primarily by its effect at the spinal cord level $(25,26)$. Furthermore, it has been shown that the inhalant agent concentration, required to induce unconsciousness and amnesia, is only $20-45 \%$ of that required to suppress purposeful movement (27). Consequently, it is possible that the horses reported here did not experience "awareness," while increase of reflex response and muscle tone were leading to the clinical assumption of a "too light" anesthesia.

Autonomic nervous responses, such as hemodynamic responses, are also commonly used as indicators of anesthetic depth and have been included in the clinical score used in the horses presently reported. However, hemodynamic responses are primarily subcortical in origin and are not depending on corticocerebral input. Therefore, they may not indicate the conscious perception of noxious stimuli (28). Additionally, they are affected by several factors, such as the pharmacodynamic properties of the different anesthetics, the duration of anesthesia, and physiologic factors. Anesthetic concentrations preventing autonomic reflexes and responses are even higher than anesthetic concentrations preventing movement (29). This corroborates the assumption that horses may still have been centrally depressed, while clinical scoring already evaluated anesthetic plane as being "too light."

The Narcotrend is primarily a monitor of the hypnotic component of anesthesia (30). Therefore, its use in horses might be questionable, because immobility is particularly mandatory for the equine surgeon, and the use of neuromuscular blocking agents is less common than in human anesthesia. However, if reliable monitoring of central depression would be available, the use of drugs with muscle relaxing properties in a multimodal anesthesia approach could reduce doses of general anesthetics and thereby their risk for side effects.

Xylazine slows the EEG in horses (31-33); therefore, the xylazine constant rate infusion used in our horses might have contributed to the fact that the NI did not increase at lower isoflurane concentrations. However, because xylazine was infused at a constant dose over a long infusion period throughout the study, we assume that it causes a continuous background dampening of the EEG. This should not completely mask a dosedependent effect of isoflurane on the EEG. When investigating EEG effects of centrally acting agents, drug administration is usually restricted to the investigated drug (34). Due to animal welfare, this was not possible in the horses reported here, because they were part of a surgical trial. Furthermore, we 
wanted to mimic clinical conditions to test the use of the NI to monitor clinical patients. Other drugs included here, as in other EEG studies, were guaiphenesin and thiopentone $(35,36)$. Guaiphenesin has been shown to have minimal influence on the equine EEG (37), and thiopentone significantly reduced the spectral edge frequency in halothane-anesthetized horses only for $10 \mathrm{~min}(38)$.

In four of the seven horses, NI began to decrease at the high ET isoflurane concentrations of 2.0 and 2.2 vol\%, respectively, which implicates that NI can distinguish a "too deep" anesthetic plane from an "adequate" plane. Isoflurane is known to suppress the EEG dose dependently and to cause burst suppression even at clinical relevant doses $(39,40)$. A special algorithm for the recognition of burst suppressions is incorporated in the Narcotrend, which leads to calculation of low indices. Therefore, the decrease of NI could be explained by an increasing amount of burst suppressions or suppression lines at high isoflurane concentrations. The clinical score assessed anesthesia as "too deep" at lower ET isoflurane concentrations, beginning from $1.6 \mathrm{vol} \%$. Therefore, the clinical advantage of NI for recognition of a deep anesthetic plane is questionable.

However, our study is not free of limitations. First, ET isoflurane concentrations used for the correlation were only indicators of effective concentrations, because equilibration phases of $15 \mathrm{~min}$ for the different end expiratory isoflurane concentrations were probably too short for equilibration of alveolar anesthetic partial pressure with arterial partial pressure and brain partial pressure. However, differences between the inspiratory and ET isoflurane concentrations were within $10 \%$ at the end of the equilibration phases, as recommended

\section{REFERENCES}

1. Grandy JL, Steffey EP, Hodgson DS, Woliner MJ. Arterial hypotension and the development of postanaesthetic myopathy in halothane anesthetized horses. Am J Vet Res (1987) 48:192-7.

2. Raisis AL. Skeletal muscle blood flow in anaesthetized horses. Part II: effects of anaesthetics and vasoactive agents. Vet Anaesth Analg (2005) 32:331-7. doi:10.1111/j.1467-2995.2005.00191.x

3. Johnston GM, Eastment JK, Wood JLN, Taylor PM. The confidential enquiry into perioperative equine fatalities (CEPEF): mortality results of phases 1 and 2. Vet Anaesth Analg (2002) 29:159-70. doi:10.1046/j.1467-2995.2002.00106.x

4. Brodbelt DC, Blissitt KJ, Hammond RA, Neath PJ, Young LE, Pfeiffer $\mathrm{DU}$, et al. The risk of death: the confidential enquiry into perioperative small animal fatalities. Vet Anaesth Analg (2008) 35:365-73. doi:10.1111/j.1467-2995.2008.00397.x

5. Otto K, Short CE. Electroencephalographic power spectrum analysis as a monitor of anesthetic depth in horses. Vet Surg (1991) 20:362-71. doi:10.1111/ j.1532-950X.1991.tb01284.x

6. Johnson CB, Young SS, Taylor PM. Analysis of the frequency spectrum of the equine electroencephalogram during halothane anaesthesia. Res Vet Sci (1994) 62:159-63. doi:10.1016/S0034-5288(97)90139-9

7. Sebel PS, Lang E, Rampil IJ, White PF, Cork R, Jopling M, et al. A multicenter study of bispectral electroencephalogram analysis for monitoring anesthetic effect. Anesth Analg (1997) 84:891-9. doi:10.1097/00000539-199704000-00035

8. Greene SA, Tranquilli WJ, Benson GJ, Grimm KA. Effect of medetomidine administration on bispectral index measurements in dogs during anesthesia with isoflurane. Am J Vet Res (2003) 64:316-20. doi:10.2460/ajvr.2003.64.316

9. Lamont LA, Greene SA, Grimm KA, Tranquilli WJ. Relationship of feline bispectral index to multiples of isoflurane minimum alveolar concentration. Comp Med (2005) 55:269-74. by Eger and Bahlman (41). Second, we used a very general clinical score modified from Hubbell and Muir to assess anesthetic depth in horses. This score might not have been ideal for a xylazine balanced inhalation anesthesia, because horses may appear more superficial than they really are, and the palpebral reflex is less dampened in an alpha 2 -agonist balanced inhalation anesthesia (42). Third, clinical usefulness of NI was investigated in a limited number of horses, and the lack of a reference method to measure unconsciousness precisely aggravates the evaluation of EEG monitors.

\section{CONCLUSION}

Correlation of NI with different ET isoflurane concentrations in horses in balanced anesthesia was poor. Common clinical parameters seemed to be more useful to ensure appropriate surgical conditions.

\section{AUTHOR CONTRIBUTIONS}

JT contributed to the design of the study, collection and analysis of the data, and prepared the manuscript. $\mathrm{KH}$ contributed to the design and collection of data. SK designed the study, analyzed the data, reviewed and finalized the manuscript.

\section{FUNDING}

The study was University funded, and no external funds were used.

10. Martín-Cancho MF, Lima JR, Luis L, Crisóstomo V, Ezquerra LJ, Carrasco MS, et al. Bispectral index, spectral edge frequency $95 \%$, and median frequency recorded for various concentrations of isoflurane and sevoflurane in pigs. Am $J$ Vet Res (2003) 64:866-73. doi:10.2460/ajvr.2003.64.866

11. Haga HA, Dolvik NI. Evaluation of the bispectral index as an indicator of degree of central nervous system depression in isoflurane-anesthetized horses. Am J Vet Res (2002) 63:438-42. doi:10.2460/ajvr.2002.63.438

12. Schultz B, Kreuer S, Wilhelm W, Grouven U, Schultz A. The Narcotrend monitor. Development and interpretation algorithms. Anaesthesist (2003) 52:1143-8. doi:10.1007/s00101-003-0603-y

13. Kreuer S, Bruhn J, Larsen R, Bialas P, Wilhelm W. Comparability of Narcotrend index and bispectral index during propofol anaesthesia. Br J Anaesth (2004) 93:235-40. doi:10.1093/bja/aeh182

14. Kreuer S, Bruhn J, Larsen R, Grundmann U, Shafer SL, Wilhelm W. Application of bispectral index and Narcotrend index to the measurement of the electroencephalographic effects of isoflurane with and without burst suppression. Anesthesiology (2004) 101:847-54. doi:10.1097/00000542-200410000-00008

15. Tünsmeyer J, Kramer S. Processed electroencephalogram monitoring of anaesthetic depth in dogs under inhalational anaesthesia. Vet Anaesth Analg (2008) 35:17. doi:10.1111/j.1467-2995.2008.00409a.x

16. Kulka AM, Otto KA, Bergfeld C, Beyerbach M, Kästner SB. Effects of isoflurane anesthesia with and without dexmedetomidine or remifentanil on quantitative electroencephalographic variables before and after nociceptive stimulation in dogs. Am J Vet Res (2012) 73:602-9. doi:10.2460/ajvr.73.5.602

17. Hubbell JA, Muir WW. Monitoring anesthesia. In: Muir WW, Hubbell JA, editors. Equine Anesthesia. St. Louis: Saunders Elsevier (2009). p. 149-70.

18. MayhewIG, WashbourneJR.Amethodofassessingauditoryandbrainstemfunction in horses. Br Vet J (1990) 146:509-18. doi:10.1016/0007-1935(90)90054-7

19. Belda E, Blissitt KJ, Duncan JC, Laredo FG, Escobar Gil de Montes M, Clutton RE. The bispectral index during recovery from halothane and 
sevoflurane anaesthesia in horses. Vet Anaesth Analg (2010) 37:25-34. doi:10.1111/j.1467-2995.2009.00507.x

20. Johnson CB, Taylor PM. Comparison of the effects of halothane, isoflurane and methoxyflurane on the electroencephalogram of the horse. Br J Anaesth (1998) 81:748-53. doi:10.1093/bja/81.5.748

21. Williams DC, Aleman MR, Brosnan RJ, Fletcher DJ, Holliday TA, Tharp B, et al. Electroencephalogram of healthy horses during inhaled anesthesia. $J$ Vet Intern Med (2016) 30:304-8. doi:10.1111/jvim.13613

22. Yamashita K, Akashi N, Katayama Y, Uchida Y, Umar MA, Itami T, et al. Evaluation of bispectral index (BIS) as an indicator of central nervous system depression in horses anesthetized with propofol. J Vet Med Sci (2009) 71:1465-71. doi:10.1292/jvms.001465

23. Rampil IJ, Laster MJ. No correlation between quantitative electroencephalographic measurements and movement response to noxious stimuli during isoflurane anesthesia in rats. Anesthesiology (1992) 77:920-5. doi:10.1097/00000542-199211000-00014

24. Rampil IJ, King BS. Volatile anesthetics depress spinal motor neurons. Anesthesiology (1996) 85:129-34. doi:10.1097/00000542-199607000-00018

25. Antognini JF, Carstens E, Buzin V. Isoflurane depresses motoneuron excitability by a direct spinal action: an F-wave study. Anesth Analg (1999) 88:681-5. doi:10.1213/00000539-199903000-00040

26. Antognini JF, Carstens E. In vivo characterization of clinical anaesthesia and its components. Br J Anaesth (2002) 89:156-66. doi:10.1093/bja/aef156

27. DwyerR,BennettHL,EgerEIII,HeilbronD.Effectsofisofluraneandnitrousoxide in subanesthetic concentrations on memory and responsiveness in volunteers. Anesthesiology (1992) 77:888-98. doi:10.1097/00000542-199211000-00009

28. Zbinden AM, Petersen-Felix S, Thomson DA. Anesthetic depth defined using multiple noxious stimuli during isoflurane/oxygen anesthesia. II. Hemodynamic responses. Anesthesiology (1994) 80:261-7. doi:10.1097/00000542-199402000-00005

29. Schmeling WT, Ganjoo P, Staunton M, Drexler C, Farber NE. Pretreatment with dexmedetomidine: altered indices of anesthetic depth for halothane in the neuraxis of cats. Anesth Analg (1999) 88:625-32. doi:10.1213/00000539-199903000-00030

30. Schmidt GN, Bischoff P, Standl T, Voigt M, Papavero L, Schulte am Esch $\mathrm{J}$. Narcotrend, bispectral index, and classical electroencephalogram variables during emergence from propofol/remifentanil anesthesia. Anesth Analg (2002) 95:1324-30. doi:10.1097/00000539-200211000-00042

31. Purohit RC, Mysinger PW, Redding RW. Effects of xylazine and ketamine hydrochloride on the electroencephalogram and the electrocardiogram in the horse. Am J Vet Res (1981) 42:615-9.

32. Mysinger PW, Redding RW, Vaughan JT, Purohit RC, Holladay JA. Electroencephalographic patterns of clinically normal, sedated, and tranquilized newborn foals and adult horses. Am J Vet Res (1985) 46:36-41.
33. Williams DC, Aleman M, Tharp B, Fletcher DJ, Kass PH, Steffey EP, et al. Qualitative and quantitative characteristics of the electroencephalogram in normal horses after sedation. J Vet Intern Med (2012) 26:645-53. doi:10.1111/j.1939-1676.2012.00921.x

34. Smith NT, Westover CJ Jr, Quinn M, Benthuysen JL, Dec Silver H, Sanford TJ Jr. An electroencephalographic comparison of alfentanil with other narcotics and with thiopental. J Clin Monit (1985) 1:236-44. doi:10.1007/BF02832817

35. Otto KA, Voight S, Piepenbrock S, Deegen E, Short CE. Differences in quantitated electroencephalographic variables during surgical stimulation of horses anesthetized with isoflurane. Vet Surg (1996) 25:249-55. doi:10.1111/j.1532950X.1996.tb01409.x

36. Murrell JC, Johnson CB, White KL, Taylor PM, Haberham ZL, WatermanPearson AE. Changes in the EEG during castration in horses and ponies anaesthetized with halothane. Vet Anaesth Analg (2003) 30:138-46. doi:10.1046/j.1467-2995.2003.00138.x

37. Johnson CB, Bloomfield M, Taylor PM. Effects of guaiphenesin on the equine electroencephalogram during anaesthesia with halothane in oxygen. Vet Anaesth Analg (2000) 27:6-12. doi:10.1046/j.1467-2995.2000.00011.x

38. Johnson CB, Bloomfield M, Taylor PM. Effects of thiopentone on the equine electroencephalogram during anaesthesia with halothane in oxygen. Vet Anaesth Analg (2000) 27:82-8. doi:10.1046/j.1467-2995.2000.00022.x

39. Eger EI II, Stevens WC, Cromwell TH. The electroencephalogram in man anesthetized with forane. Anesthesiology (1971) 35:504-8. doi:10.1097/00000542-197111000-00010

40. Murrell JC, Waters D, Johnson CB. Comparative effects of halothane, isoflurane, sevoflurane and desflurane on the electroencephalogram of the rat. Lab Anim (2008) 42:161-70. doi:10.1258/la.2007.06019e

41. Eger EI II, Bahlman SH. Is the end-tidal anesthetic partial pressure an accurate measure of the arterial anesthetic partial pressure? Anesthesiology (1971) 35:301-3. doi:10.1097/00000542-197109000-00013

42. Kalchofner KS, Ringer SK, Boller J, Kästner SBR, Lischer C, BettschartWolfensberger R. Clinical assessment of anesthesia with isoflurane and medetomidine in 300 equidae. Pferdeheilkunde (2006) 22:301-8.

Conflict of Interest Statement: The authors declare that the research was conducted in the absence of any commercial or financial relationships that could be construed as a potential conflict of interest.

Copyright $\odot 2016$ Tünsmeyer, Hopster and Kästner. This is an open-access article distributed under the terms of the Creative Commons Attribution License (CC BY). The use, distribution or reproduction in other forums is permitted, provided the original author(s) or licensor are credited and that the original publication in this journal is cited, in accordance with accepted academic practice. No use, distribution or reproduction is permitted which does not comply with these terms. 